\title{
A Volunteer Alcohol Consumption Reduction Campaign: Participatory Action Research among Thai Women in the Isaan Region
}

\author{
Darunee Jongudomkarn
}

\begin{abstract}
Cancer is one of the leading causes of death in Thailand as well as other countries. Evidences from studies in Asia show that alcohol consumption has been linked to an increased risk for various types of cancer. In the qualitative participatory action research presented here, the possibilities of a volunteer women's movement to reduce harmful alcohol consumption by facilitating the creation of emancipatory knowledge in the Thai-Isaan community was explored. During in-depth interviews with 10 female volunteer participants, the following themes emerged: merit making, developing a sense of personal empowerment, and the experience of participating in an action plan that utilized various strategies. The project activities empowered participants to create and share knowledge which was then applied toward action for change. Adoption of this type of program by local health care providers to reduce health problems and risks related to alcohol misuse in similar contexts and cultures is recommended.
\end{abstract}

Keywords: Women's movement - participatory action - Thailand - alcohol consumption reduction - cancer prevention

Asian Pac J Cancer Prev, 15 (17), 7343-7350

\section{Introduction}

Much research has been conducted on the issue of alcohol-related mortality and morbidity around the world (WHO, 2010). Diseases and injuries attributed to alcohol kill millions and harm tens of millions of people each year worldwide. Based on extensive reviews of research studies, there is a strong scientific consensus of an association between alcohol drinking and several types of cancer (IARC Working Group on the Evaluation of Carcinogenic Risks to Humans, 2010; 2012). The results of meta-analysis published in English in the last 10 years searching in the PubMed database, indicate that moderate to heavy consumption of alcohol increases the risk of developing cancer of the oral cavity and pharynx, esophagus, stomach, larynx, colorectum, central nervous system, pancreas, breast and prostate. It is also observed that alcohol consumption may be inversely related to thyroid cancer (de Menezes et al., 2013). In addition, the meta-analysis study also showed a dose-response relationship of alcohol consumption with breast cancer mortality and recurrence and alcohol consumption of $>20$ $\mathrm{g} / \mathrm{d}$ was associated with increased breast cancer mortality (Gou et al., 2013). The literature reviews on link between alcohol and cancer in the Asia Pacific region revealed that alcohol drinking modifies the risk of cancers and other diseases. For instance, in a Korean-population- based cohort study, the odds ratio for developing distal stomach cancer for those who consumed more than $15 \mathrm{~g}$ of alcohol a day was 1.2 (95\%CI 1.0-1.3) compared with non-drinkers (Sung et al., 2007). In the Japanese cohort study with a 20-year follow-up period, increased cancer mortality risks were observed, especially for people who both started smoking early and drank alcohol (Yaegashi et al., 2014). The case-control study report from China found that increased the colorectal cancer susceptibility was altered by and cancer risk due to high alcohol intake (Gao et al., 2013). In addition, evidence study report from China showed that alcohol drinking and tobacco smoking were responsible for $86 \%$ of liver cancer mortality and incidence in 2005 (Fan et al., 2013). While the results of systematic reviews to evaluate associations between tea consumption, alcohol drinking and physical activity and breast cancer risk among Chinese females were found that tea consumption and physical activity were significantly associated with a decreased risk of breast cancer (Gao et al., 2013). Thus, many countries' governments around the world included Thailand's are committed to reducing harms caused by alcohol (WHO, 2010).

Excessive alcohol consumption in Thailand has been steadily increasing in severity, leading not only to personal problems but also adversely affecting the family, community and society. Research indicates that this situation is related to the way people have been 
socialized, resulting in a lack of knowledge and awareness of the health risks involved (CAS Board, 2014). Cancer is an important cause of illness and mortality in Thailand and around the world (WHO, 2010). However, cancer incidence varies widely. It was found that liver and bile duct cancer is the most common cancer in Thailand, while it is not among the 10 most incident cancers in the United States. Moreover, alcohol overconsumption and Cirrhosis are possible risk factors and causes of liver cancer and bile duct cancer (Jung et al., 2014; Subimerb et al., 2014).

In Thailand, the number of male and female teenage drinkers has increased, while the average 'first drink' age has decreased. Recent statistics indicate that among those 15-24 years of age the rate of alcohol consumption is $23.7 \%$, with an average 'first drink' age of 17.2 years. For the 25-59-year-old age group the rate of alcohol consumption is $37.3 \%$, with an average 'first drink' age of 20.6 years. Elderly Thais, 60 years and older, still exhibit a high rate of alcohol consumption (16.6\%). Overall, the average age of first consumption is 20.5 years: males begin drinking alcohol earlier (age 19.4 years), while females start to drink at an average age of 24.6 years (National Statistical Office, 2014).

Alcohol consumption poses significant social and medical problems in Thailand and many parts of Asia. It directly affects work efficiency, mental and physical health, emotional well-being, family relationships, child development, crime and accident rates, the prevalence of chronic diseases, and ultimately the national economy (WHO, 2010; Wright and Leahey, 2009), causing a burden on society from increased expenditures on healthrelated issues and disability (Choi et al., 2010). Women are socialized to be the caretakers of ill family members. Typically they not only have a career outside the home, but continue to juggle traditional household responsibilities after-hours. Much female suffering is caused by struggling to achieve the "male standard" at work, while meanwhile trying to maintain the standards of being a perfect wife and mother at home (Hild, 2012). Thus, women are particularly subject to the stress of family members' alcohol misuse; they are preoccupied with their family's needs, and consequently have little time to attend to their own needs, as well as their health. Therefore, encouraging women to be change agents through active participation in health programs and research could empower and benefit them in their role as key family caregivers (Unicef, 2014).

Hence, the main purpose of the present paper was to explore the possibilities of initiating a Thai-Isaan women's volunteer health movement to reduce harmful alcohol consumption in the community and to facilitate the creation of emancipatory knowledge as well as a more thorough understanding regarding the reduction of harmful alcohol consumption practices. The women participating in this study performed their role as alcohol committee volunteers by campaigning to promote responsible consumption and to prevent alcohol healthrelated problems.

The study was guided by critical social theory (Ganann, 2013), and the research model was participatory action research (PAR). PAR refers to a process whereby the researchers and stakeholders collaborate in the design and conduct all phases of the research process (MacDonald, 2012). The recognition of inequitable relationships between researchers and participants has previously emerged in studies conducted in the developing world. Participatory action research helps diminish the distinctions inherent in this traditional relationship (Ganann, 2013), and thus was considered to be an appropriate approach for the present study. PAR implies a process of planning, evaluation and research. Accordingly, this study encouraged participants to join in with planning, executing, evaluating, and sharing accountability throughout the study.

Based on Thai cultural traditions, the women of Isaan generally have been raised in a social system that discourages personal autonomy. Their world primarily revolves around their husbands and families. Thai cultural social values and norms determine the roles of women in familial relationships, as do Buddhist beliefs (Yong et al., 2013). The family system in Thailand is still strong, and in general women have a key role to play in sustaining the family and in caring for ill members. Thus, as the family members responsible for family health care provision, the study participants had personally suffered experiences related to alcohol consumption problems.

The women participants were involved in the entire research process. After the conclusion of the project, these women became resource persons responsible for alcohol consumption campaigns in the community and nearby areas.

\section{Materials and Methods}

This study was part of a larger research project based on participatory action research, which was initiated by community members via a community forum. The study was approved by the Khon Kaen University Ethics Committee for Human Research, Khon Kaen, Thailand. Consent was acquired from individuals for interviews and home visits. Data collection was carried out from January 2012 through January 2013. Participation in the study was voluntary. The ten women who were recruited were nominated via a community forum.

The data were collected through individual and group interviews and discussions. Each interview was soundrecorded and transcribed. Open-ended questions were utilized to elicit a deeper and comprehensive view of the participants' experiences of reducing health risks relating to alcohol consumption among their neighbors and family members.

Through a reciprocal learning process, the participants' knowledge pertaining to health risks related to alcohol consumption in their cultural context was acknowledged. Data management was completed manually by the author. Analysis and interpretation of the data were presented to the participants as well as the research assistant, who then validated the accuracy of the analysis and interpretation of the findings.

Defining the roles of the research team and participants was the first step of the process. Sharing knowledge and power in addition to decision making were keys to research actions. This vital interaction helped to enhance 
the potential and capacities of all participants. To ensure the quality of research results, guidelines of research validity and reliability were proposed to all members. Participatory training workshops for the women who were nominated by the community were conducted by the team, focusing on knowledge concerning harmful alcohol consumption, gender issues amid consumption, strategic planning, and family care. The participants then developed a strategic plan and an action plan, together with a continuing evaluation process.

\section{Participants, setting, beliefs, and alcohol consumption}

The participants were 10 female health volunteers aged 39-48 years. All spoke the Isaan dialect and were married. Five were farmers, four were vendors, and one was a laborer. Most were living with their adult children and their families. The participating community was located in semi-urban northeast Thailand.

Thailand, which is situated in Southeast Asia, covers an area of $513,115 \mathrm{~km} 2$. It is divided into 76 provinces and 6 regions. The Isaan (northeastern) region of Thailand was the setting for this study. With an area of approximately $168,854 \mathrm{~km} 2$, Isaan constitutes roughly one-third of the entire area of the country (National Statistic Office, 2014). There are 20 provinces within Isaan, which is bordered to the north and east by the Mekong River and to the south by Cambodia. Occupations throughout the region vary from poor laborers to government officials and business owners. Natural reservoirs in the north serve as popular recreational areas. Stalls and businesses selling food and alcohol are omnipresent.

Theravada Buddhism is the dominant religion. Approximately $95 \%$ of the population in Thailand declare themselves to be Buddhist (National Statistic Office, 2014). However, the inhabitants of the Isaan region have their own distinct cultural identity and beliefs. Many rituals are concerned with ancestor worship, and often involve alcohol in some way. Most lay persons in northeastern Thailand also believe in the supernatural, as evidenced by a widespread belief in magical amulets, spirits, ghosts, divine incarnations, and mystical incantations. Nevertheless, these supernatural beliefs are not part of Buddhist teachings (Jongudomkarn et al., 2012).

Female participants in this study had generally completed primary-level schooling. Although they had the dual role of home providers and wage earners, they were nevertheless nominated by the community forum to be health volunteers for the alcohol consumption reducing campaign project.

Traditionally, women are loyal to both their birth family and, after marriage, their family of procreation. To her biological family a woman is a daughter, an older sister, or a younger sister. In the relationship between a woman and her family of procreation, a woman plays the role of wife, mother and grandmother. Buddhism teaches that to be born a woman, one has less merit than a man. Peasants said that in this life if you are a woman you should make a lot of merit so that you will be born a man in the next life. (Sohm-In, 1994).

At the first session the project objectives and the research process were explained. The roles of team members and of women participants in the PAR process were described, and it was ensured that all participants had the capability of being effective partners in the research. The local family nurses, the research assistants, assumed responsibility for facilitating the focus group sessions; and campaign for responsible alcohol consumption and prevention of alcohol-related health risks to members of the community. The participants expressed their commitment to working together for change in the community.

\section{Data collection}

Qualitative data was collected through observation, interviews, and focus group sessions. Critical conversation was used as a tool to produce research-focused knowledge. In addition, conversation also helped provide a better understanding of the women participants' worldviews, lives and experiences (MacDonald, 2012). Two research assistants served as facilitators for the continuing focus group discussions and for interviews with the 10 participants. The author attended all sessions, which were carried out at the local community center over a period of one year. Forty sessions were conducted for approximately 2 hours each, using the Isaan dialect. Demographic data were collected at the first session, which began with the following sample questions:

"Can you tell me the reasons you wish to be a volunteer?" "What effect does heavy alcohol consumption have on you, your family, and community?" "What does it mean to you to be active in the group?" "What is the process developed and adopted by women participants to promote family and community members' health and well-being?" "What have you learned through your volunteer work?"

Every session was tape-recorded; detailed notes of important issues, with typical examples, were handwritten for use in all subsequent sessions (Yanow and SchwartzShea, 2013)

\section{Data analysis}

Content analyses were used for interpreting qualitative data. These approaches included examining the meaning of the content of discussions. During the group process, the research team encouraged idea production among the participants, whose explanations and interpretations led the group to greater insights (Streubert and Carpenter, 2011).

The study rigor ensured that the findings accurately reflected the participant's experiences. Trustworthiness criteria, including credibility, dependability, transferability and conformability, were used to establish and document rigor in this study (Streubert and Carpenter, 2011). To provide evidence of credibility we used several techniques. Prolonged engagement involved spending time with the subject matter to develop an understanding of the context of the experience (Yanow and Schwartz-Shea, 2013). Dependability required the researcher to provide enough information to allow other researchers to follow the development of the study. The author observed every focus group discussion and supervised throughout the transcription process. The research assistants recorded 
observational data for each participant and transcribed discussions. The quality of discussions and transcriptions was ensured and evaluated by the research team. Thick description was used to establish transferability or fittingness. Member check, to help improve the accuracy, credibility and transferability of the study, was an additional technique used by the team. At the end of each session, the notes were reviewed with the participants to check for completeness and accuracy.

The research team reported on the outcomes of the preand post-intervention scores of the participating community members on the Alcohol Use Disorder Identification Test (AUDIT) (Silapakij and Kittirattanapaibul, 2009), developed by the World Health Organization. Reflections on the research process and results were obtained through group discussions.

\section{Results}

Participants described their experiences of suffering caused by family members who were alcoholics. This inspired them to get involved in the project, with the additional goal of making merit. During the course of the project, they not only made connections but also developed relationships. They gained knowledge and skills as well as increased self-esteem and confidence to help others and reduce alcohol consumption problems in their communities. At the individual, family and community level, well-being was enhanced through women's community volunteerism.

To achieve the objectives of the study, it was necessary to gain an understanding of the perceived psychosocial and personal benefits of the women participating as key actors in the project. Two themes that everyone agreed upon were: (a) the opportunity of making merit, and (b) experiencing personal empowerment.

Regarding identifying strategies for change and proposed action plans, it was decided by participants in the course of their efforts to take care of their family members' and neighbor's health as well as to reduce these people's health risks related to alcohol consumption. Action plans were formulated, including: (a) workshops for the female participants; (b) community campaign activities for alcohol consumers, conducted by the women participants; (c) weekly home visits for the purpose of family meetings and earnest discussions about responsible alcohol consumption; (d) sharing the project findings with the larger community via a community forum; and (e) various other forms of responsible alcohol consumption campaigns.

\section{Merit making}

Buddhist teachings encourage the practice of religious rituals in order to achieve the following: (1) merit which would bring about good fortune in the future or in the next life; (2) a miracle which would free one from poverty and stress; and (3) comforting oneself with religious principles. Participants perceived that getting involved with the project to decrease alcohol consumption problems was a form of merit-making. Alcohol, according to Buddhist teaching, is a poison. So if they could assist others by helping people stop consuming this poison, they would be making merit and thereby gain spiritual rewards in the future. In addition, Buddhist teaching emphasizes that women who are mothers should sacrifice their personal happiness for the family's happiness. This concept has always been taught to Thai women, who are deeply affected by it.

Throughout the process we could observe that the participants consistently described how they placed their family's needs and societal expectations before their own. One participant said: "I am so proud to have done something for the good of our small society. I can see happiness occurring in a family whose members can reduce their alcohol consumption. We are acting as gatekeepers, and are keeping an eye on problem drinkers who are members of our community. We are neighbors so I can lend a helping hand to this; it's a form of meritmaking.” (Ms. J)

\section{Experiencing personal empowerment}

A primary feedback from the women participating in the research project was a sense of personal empowerment. They described an increased knowledge concerning harmful alcohol consumption, and an improvement in their people skills as a result of nurturing and taking care of their own as well as other families in the community in their role as activist health promoters. They also gained experience from the community campaign, including a feeling of reciprocity with community members and the satisfaction of creating social change.

The female participants identified numerous personal benefits gained from their volunteer involvement, including increased self-esteem, enhanced social skills, family caring skills, and a sense of purpose. Some women participants had a chance to tell their story of addressing alcohol problems on local TV, while others received awards from national agencies in recognition of their contributions.

The following are sample quotes from some of the women participants, expressing their feelings about the project and its impact on their lives:

"I was a shy person before joining the project....Yes, the process of this project has made my self-esteem go up. It's made me realize that I can do things: I can better my input...." (Ms. M)

"I enjoy working with the kids whose parents go out from the community to work as vendors; I love doing it. They call me a 'second mom' with their smiling faces when they see me in public places. It makes me feel good; I seem to be somebody in this community." (Ms. S)

"As a participant in this project it's helped me to learn how to deal with my family and with teenagers in the community about reducing harmful alcohol consumption. They had a lot of problems; some of them used to lie, used to steal.... A benefit of the project is that it's helped me to better understand and approach these teenage drinkers, as well as my own children. In discussing this point with other parents and by changing our roles towards kids, we can see that we've become closer. We now spend a lot of time with these kids, and they're a lot happier and friendlier." (Ms. W) 


\section{Action plans}

One of the goals of the project was to support the women as key actors in promoting the education of family members and the community about alcohol abuse, and in mobilizing them for action toward responsible alcohol consumption. Over a period of one year, the following multilevel interventions were planned and performed by the ten female participants: (a) workshops for the participants during the first month; (b) monthly community campaign activities for participating alcohol consumers for ten months, beginning after the second month; (c) weekly home visits for family meetings and conversations on responsible alcohol consumption; (d) monthly sharing of project findings with the larger community via community forums; and (e) various other forms of responsible alcohol consumption campaigns.

The plan was put into action and all ten participants and the researchers participated in every intervention session. The education campaign interventions were conducted on the basis of possible consequences for individuals, families and society from alcohol misuse, and emphasized the positive gains that could accrue from reducing consumption. Via the series of community forums, the community proposed its own suggested guidelines for alcohol consumption, such as no alcohol sales on Buddhist holy days, no alcohol sales to persons under 18 years of age, and encouraging abstinence during festivals.

\section{Workshops for female participants}

The participants and researcher team worked together to plan, organize, promote and conduct training workshops for female participants. The workshops focused on responsible alcohol consumption, and also on gender issues in relation to consumption due to social constructs and norms. For example, some males may say to themselves: "I drink, therefore I'm a man". At the same time, some women may drink to show their gender equality as a 'smart working woman'. Thus, consumption of alcohol is clearly negatively promoted by gender stereotypes (WHO, 2005), a factor which should not be overlooked when addressing the problem.

The participants developed a two-phase action plan, as follows. For the first phase, the participating individuals were divided into areas of responsibility covering the community, meaning that they were accountable for campaigning and taking care of families in specified areas. At the same time they collaborated with community leaders and the researchers in conducting a community forum to create suggested alcohol guidelines for the community. The second phase focused on early detection of high-risk consumption; participants were facilitated by local nurses who screened lay people at high risk of alcohol dependency. At-risk drinkers were identified using AUDIT and were recruited to join a "Stop Consumption Project". Continued home visits and in-depth dialogue with consumers, facilitated by the researchers, were key tasks to be performed by the female participants in their respective zones of responsibility. Street campaigns. The creation and preparation of street campaigns was carried out by the participants in conjunction with community leaders and researchers. These campaigns presented information showing the negative consequences to individuals, families and society as a result of irresponsible alcohol consumption, and how these consequences could be reversed, as well as the positive results that could be obtained from responsible consumption. The campaigns were conducted every two months on Buddhist holy days, in the evening when most people were at home. Each implementation of the street campaign was preceded by a parade of community leaders and their associates, which included local school students and health volunteers, moving through the community's communal areas.

Poster campaign: a poster campaign for responsible alcohol consumption was launched, placing them in community public areas. Using colloquial language, the posters highlighted how alcohol produces negative consequences on the human body, economy and society, and advised people to moderate their consumption. The poster also contained a local poem that was familiar to members of the community.

Leaflets: the leaflet content covered alcoholconsumption patterns, the consequences, and when people should seek medical advice. Leaflets were hand-delivered to each household at the time of the street campaigns, as well as during the home visits. The message focused on the signs and symptoms of alcohol dependence and the ways to decrease consumption. Leaflet content was designed by the researchers, and was revised by five local activist scholars.

\section{Weekly home visits}

Individuals in the community who were identified as high-risk drinkers by the local health center were asked to participate in the project by means of receiving weekly home visits by the participants and researchers. The names of ten drinkers ( 7 males and 3 females) were chosen by randomly drawing lots. One female participant was assigned to each. Most visits took place at the family's homes, which allowed all family members to meet with the female participant along with the assigned researcher. The family visits contributing to the responsible alcohol consumption educational program were spread over twelve different sessions. In addition, meetings via telephone were conducted if requested due to certain situations.

The family visit module was developed by the research team and the assigned participants; this included information on high-risk consumption, responsible alcohol consumption, and lessons learned from personal experiences. Examples of the topics discussed included the influence of peer pressure on consumption, and alcohol's effects on a drinker's finances, family relationships, and health. Meanwhile, each session integrated the concept of gender issues into the conversations. Interview feedback indicated that the family meetings helped to develop better understanding among drinkers regarding their family's suffering.

The 10-item Alcohol Use Disorder Identification Test (AUDIT), which is considered to be an efficient means of 
early identification of problem consumption, was used in this study. The instrument covers the domains of alcohol consumption, consumption behavior, and alcohol-related problems. It was originally designed by the World Health Organization to screen for hazardous alcohol intake in primary health care settings. Test scores of participants at pre- and post-intervention were recorded. At the end of the 12-month program, it was found that each subject displayed differing AUDIT points between the first and final dates of intervention. Verbal feedback from the alcohol-consuming attendees on their AUDIT scores was positive.

During home visits, family members freely communicated their feelings to one another. The following are illustrative excerpts from the conversations. "Every time my son leaves home with his motorcycle, I feel very unhappy. I can't sleep all night because of concerns that he will get drunk and have an accident." Children also expressed their feelings concerning their fathers' alcohol dependence. "I do not like my dad's consumption. He is always drunk, and seems out of control. Ifeel ashamed to be a drunkard's son." Comments from participants who were alcohol drinkers included: "We just deeply understand our family's feeling when we have opportunities to share our experiences in the group discussions." As one member said: "I've got my family back because we now have the chance to share our feelings. Beforehand we always had conflict with the issues of alcohol consumption in our family; we've learned how to communicate and keep good relationships going in our family. This will benefit us in keeping better communication among family members." When asked how useful the project had been, one father replied: "I think it has been a great success." Continued action projects for responsible alcohol consumption in the community were suggested to be maintained.

\section{Sharing of findings with community members}

Community forums were arranged for the women participants and researchers to share their findings and insights with community members. The monthly events took place at the community meeting hall. Approximately 100 participants attended each time over the course of ten forums. One of the female participants conducted the forums, which included sharing the findings from conversations during home visits, imparting information about the adverse effects of excessive alcohol consumption, and encouraging members of the community to make a commitment to responsible alcohol consumption. There were ample opportunities for all participants to share their experiences and concerns, and to build a consensus for future community health promotions.

\section{Discussion}

This community-based responsible alcohol consumption campaign was the first of its kind in Thailand, in that it was conducted by adult female community volunteers. Our findings suggest the potential of participatory action research as an effective approach to creating health educational knowledge and empowering participants to be actors in reducing health risks in their own community. The concept of empowering people in their own care is supported in the WHO Declaration of Alma Ata (WHO, 2000), which found that PAR is associated with evidence of high community acceptance -which in this instance represents significant sustained change in consumption patterns in the participating community. Two key themes were unanimously agreed upon by the women participants concerning their experiences as community volunteers for reducing harmful alcohol consumption: (a) the opportunity to make merit, and (b) experiencing personal empowerment.

These themes are consistent with previous studies (Lundberg and Thrakul , 2013; Tuicomepee et al., 2012) indicating that Thai women place importance on meritmaking, which is a key concept in Buddhism. The merit that accumulates as a result of good deeds, acts or thoughts will carry over throughout one's life or in subsequent incarnations. In addition, from the Thai viewpoint, Buddhism implies that being born a woman, one possesses less merit than a man (Kabinlasingh, 1984).

A critical key to the success of this study was empowering the women participants and giving them an opportunity to become partners in a health promotion project via PAR within the context of reducing alcohol consumption (Kral et al., 2011). Our findings suggest that women's empowerment is enhanced by the process of PAR, producing positive consequences of increased self-esteem (Ozer and Douglas, 2013). Furthermore, the opportunity of working collaboratively is seen as a cornerstone of empowerment. Female empowerment and gender issues in relation to alcohol consumption were an explicit concern throughout this research. The concepts of gender equality and alcohol consumption were also key issues to be discussed in training workshops for the female participants, in addition to responsible alcohol consumption campaigns for community members.

The research also focused on strengthening the importance of the participants' health information and family approach through capacity and confidence-building skills and assertiveness training. As participants, the women gained access to useful information and then transmitted this information to others - processes that were vital to community development and promoting changes in harmful alcohol consumption. By learning the important issues, they were able to work effectively with at-risk families and children. Accordingly, the female participants acted as health information and knowledge mediators.

Regarding identifying strategies for change and action plans decided by the women participants in their efforts to take care of their family members' and neighbor's health as well as reducing these people's health risks related to alcohol consumption, action plans were conducted, including workshops, community campaigns in various forms, continuing home visits, and the sharing of project findings with the larger community via community forums. Bringing together findings gave empirical evidence of responsible consumption campaigns in the community to some of the characteristics related to stakeholder engagement for empowerment and capacity building. The success of participatory approaches is interdependent with the persons together with families 
engaged thereby highlighting the importance of women's roles and personal contacts.

The women participants conducted meetings with families suffering from irresponsible alcohol consumption problems, using techniques of tailored deep discussion. Training the participants to create an atmosphere conducive to sharing information and feelings among family members helped to foster closer relationships (Wright and Leahey, 2009). Using the trust that comes with being neighbors, the women participants, professional family nurses and researchers collaborated to conduct these meetings, which helped team members develop a deeper understanding of relationship issues and relate better to a family member's alcohol consumption problem. Moreover, family meetings also address the needs of the family when one family member is suffering from an issue that alters his or her behavior or habits in negative ways. Sharing experiences with community members via community forums and campaigns gave the members a sense of collective identity.

These outcomes demonstrate the success of supporting women's groups and creating new change agents for enhancement of individual, family and community health. The findings demonstrated that these actions should be adopted globally by local primary health care teams to reduce health problems and risks related to alcohol misuse in similar contexts and cultures, particularly in the context of Asia.

Although this participatory action research study was conducted in a real community context, there were a number of unique perspectives: in particular, Thai culture. According to Thai culture and tradition, close relationships in the community are fundamental. This is somewhat different from Western cultures, where people typically regard alcohol consumption as one's own personal business. Therefore, community-based participatory responsible alcohol consumption campaigns and the creation of consumption guidelines might be considered a violation of personal rights.

This study adopted participatory action research (PAR) to reduce the health risks related to harmful alcohol consumption by community members. It was based on reflection, data collection, and action through involving other people who, in turn, took action to improve their own and their neighbors' health. This study demonstrated changes among the women participants, individuals and families by way of participatory action research. Home visits, together with family meetings by the female participants facilitated by a local nurse, were found to be instrumental in bringing about closer family member relationships. Delegation and assignment of community area responsibilities for women participants to campaign for responsible alcohol consumption, and in the process inspire community collective action, were a key success strategy of the project. The findings indicate that sharing data findings with community members through community forums enabled members to have a feeling of empowerment by identifying problems, joining in with planning, and facilitating changes in behavior. Finally, with the ongoing support of the community members, the local health service unit and the local governor, the continuation of meaningful action was agreed upon. The author believed that the lessons learned and valuable experiences from this study would benefit those who share a similar problem, as well as a similar culture and context.

\section{Acknowledgements}

The author is obliged to the Center of Alcohol Studies (CAS) of Thailand and the Center for Research and Training on Gender and Women's Health (CRTGWH), Khon Kaen University, for providing research funding. My greatest thanks go to the women who shared their valuable experiences. I also would like to acknowledge the dedication and inspiring work of the research assistants, Ms. Samran Pulthong, Ms. Khanittha Pongsiri, Ms. Puanpetch Singhawara, and Ms. Patama Chawalison. The author declared no potential conflicts of interest with respect to the research, authorship, and/or publication of this article.

\section{References}

Center for Alcohol Studies (CAS) Board. (2014). Status of alcohol consumption. Office of Narcotics Control Board, Bangkok [in Thai].

Choi J, Miyashita M, Hirai K et al (2010) Preference of place for end-of-life cancer care and death among bereaved Japanese families who experienced home hospice care and death of a loved one. Support Care Cancer, 18, 1445-53.

de Menezes RF, Bergmann A, Thuler LCS (2013). Alcohol consumption and risk of cancer: a systematic literature review. Asian Pac J Cancer Prev, 14, 4965-72.

Fan J-H, Wang J-B, Jiang Y, et al (2013). Attributable causes of liver cancer mortality and incidence in China. Asian Pac J Cancer Prev, 14, 7251-6.

Ganann R. (2013). Opportunities and challenges associated with engaging immigrant women in participatory action research. J Immigrant Minority Health, 15, 341-9.

Gao C-M, Ding J-H, Li S-P, et al (2013). Polymorphisms in $\mathrm{XRCC} 1$ gene, alcohol drinking, and risk of colorectal cancer: a case-control study in Jiangsu province of China. Asian Pac J Cancer Prev, 14, 6613-8.

Gao Y, Huang YB, Liu XO, et al (2013). Tea consumption, alcohol drinking and physical activity associations with breast cancer risk among Chinese females: a systematic review and meta-analysis. Asian Pac J Cancer Prev, 14, 7543-50.

Gou Y-J, Xie D-X, Yang K-H, et al (2013). Alcohol consumption and breast cancer survival: a meta-analysis of cohort studies. Asian Pac J Cancer Prev, 14, 4785-90.

Hild L. (2012). The Woman question in medicine: an update. The Hastings Center Report, 42, 38-45.

IARC Working Group on the Evaluation of Carcinogenic Risks to Humans. (2010). Alcohol consumption and ethyl carbamate. IARC Monographs on the Evaluation of Carcinogenic Risks in Humans, 96, 1383.

IARC Working Group on the Evaluation of Carcinogenic Risks to Humans. (2012). Personal habits and indoor combustions. Volume $100 \mathrm{E}$. A review of human carcinogens. IARC Monographs on the Evaluation of Carcinogenic Risks in Humans, 100, 373-472.

Jongudomkarn D, Forgeron P, Siripul P, Finley A. (2012). My child you must have patience and Kreng Jai: Thai parents and child pain. J Nurs Scholarsh. 44, 323-331.

Jung SH, Gombojav B, Park E-C, et al (2014). Population 
based study of the association between binge drinking and mortality from cancer of oropharynx and esophagus in Korean men: the Kangwha Cohort Study. Asian Pac J Cancer Prev, 15, 3675-9.

Kabinlasingh C. (1984). Buddhism and the status of women. In 'Buddhism and society in Thailand', ed Terwiel R. Southeast Asia Review Office, Ranchai, India.

Kral MJ, Ramírez García JI, Aber MS, et al. (2011). Culture and community psychology: toward a renewed and reimagined version. Am J Community Psychol, 47, 46-57.

Lundberg PC, Thrakul S. (2013). Religion and self-management of Thai Buddhist and Muslim women with type 2 diabetes. J Clin Nurs, 22, 1907-16.

MacDonald C. (2012). Understanding participatory action research: a qualitative research methodology option. CJAR, 13, 34-50.

National Statistic Office. (2014). Population characteristics. The Office of National Statistic Department, Bangkok [In Thai].

Ozer EJ, Douglas L. (2013). The Impact of Participatory Research on Urban Teens: AnExperimental Evaluation. Am J Community Psychol, 51, 66-75.

Silapakij P, Kittirattanapaibul P. (2009). AUDIT. WHO, CAS, Thailand Minister of Public Health Office, Bangkok [In Thai].

Sohm-In A. (1994). World visions of Isaan people. University of Srinakarintaravirot Press, Mahasarakham [In Thai].

Streubert HJ, Carpenter DR. (2011). Qualitative research in nursing: Advancing the humanistic imperative, 5th ed, J.B. Lippincott Company, Philadelphia.

Sung NY, Choi KS, Park EC, et al (2007) Smoking, alcohol and gastric cancer risk in Korean men: the National Health Insurance Corporation Study. Br J Cancer, 97, 700-4.

Tuicomepee A, Romano JL, Pokaeo S, etal (2012). Counseling in Thailand: Development from a Buddhist Perspective. $J C D, 90,357-61$.

Unicef. (2014). Women as Changes of Agents. Retrieved May 21, 2014,

World Health Organization (WHO). (2000). The family health nurse: context, conceptual,

Framework, and curriculum. WHO Regional Office for Europe, Copenhagen.

World Health Organization (WHO). (2005). Alcohol, gender, and drinking problems. WHO Press, Geneva.

World Health Organization (WHO). (2010).World Health Statistics 2010. WHO Press, Geneva.

Wright LM, Leahey M. (2009). Nurses and families: a guide to family assessment and intervention. F.A. Davis, Philadelphia.

Yaegashi Y, Onoda T, Morioka S, et al (2014). Joint effects of smoking and alcohol drinking on esophageal cancer mortality in Japanese men: findings from the Japan collaborative Cohort Study. Asian Pac J Cancer Prev, 15, 1023-9.

Yanow D, Schwartz-Shea P (2013). Interpretation and Method: Empirical research and the interpretative turn. 2nd ed, ME. Sharpe, Inc., New York.

Yong H-H, Savvas S, Borland R et al (2013). Secular versus religious norms against smoking: which is more important as a driver of quitting behavior among Muslim Malaysian and Buddhist Thai smokers? Int J Behav Med, 20, 252-8. 